\title{
molecules
}

ISSN 1420-3049

(C) 2007 by MDPI

www.mdpi.org/molecules

\author{
Full Paper
}

\section{(-)-Catechin in Cocoa and Chocolate: Occurence and Analysis of an Atypical Flavan-3-ol Enantiomer}

\author{
Michael Kofink*, Menelaos Papagiannopoulos and Rudolf Galensa
}

University of Bonn, Department of Nutrition and Food Sciences, Research Area: Food Chemistry I, Endenicher Allee 11-13, 53115 Bonn, Germany.

* Author to whom correspondence should be addressed: e-mail: m.kofink@uni-bonn.de, Tel.: +49-228-73-7865, Fax: +49-228-73-3757

Received: 30 May 2007; in revised form: 2 July 2007 / Accepted: 2 July 2007 / Published: 4 July 2007

\begin{abstract}
Cocoa contains high levels of different flavonoids. In the present study, the enantioseparation of catechin and epicatechin in cocoa and cocoa products by chiral capillary electrophoresis (CCE) was performed. A baseline separation of the catechin and epicatechin enantiomers was achieved by using $0.1 \mathrm{~mol} \cdot \mathrm{L}^{-1}$ borate buffer $(\mathrm{pH} 8.5)$ with $12 \mathrm{mmol} \cdot \mathrm{L}^{-1}$ (2-hydroxypropyl)- $\gamma$-cyclodextrin as chiral selector, a fused-silica capillary with $50 \mathrm{~cm}$ effective length (75 $\mu \mathrm{m}$ I.D.), $+18 \mathrm{kV}$ applied voltage, a temperature of $20{ }^{\circ} \mathrm{C}$ and direct UV detection at $280 \mathrm{~nm}$. To avoid comigration or coelution of other similar substances, the flavan-3-ols were isolated and purified using polyamide-solid-phaseextraction and LC-MS analysis. As expected, we found (-)-epicatechin and (+)-catechin in unfermented, dried, unroasted cocoa beans. In contrast, roasted cocoa beans and cocoa products additionally contained the atypical flavan-3-ol (-)-catechin. This is generally formed during the manufacturing process by an epimerization which converts (-)-epicatechin to its epimer (-)-catechin. High temperatures during the cocoa bean roasting process and particularly the alkalization of the cocoa powder are the main factors inducing the epimerization reaction. In addition to the analysis of cocoa and cocoa products, peak ratios were calculated for a better differentiation of the cocoa products.
\end{abstract}

Keywords: Flavonoids; Cocoa; Chocolate; Catechin; Epicatechin; Epimerization; Enantioseparation; Capillary Electrophoresis; LC-MS; Isolation; Purification 


\section{Introduction}

In recent years the analysis of polyphenolic compounds in raw and processed food became more important with regard to their numerous physiological properties [1-3]. Due to their various effects, the flavan-3-ols group is of major interest. In plants they can act as chemical signals to attract or deter insects and provide a defense against pathogens and environmental stress [4]. Regarding their health benefits in humans, they show positive cardiovascular, anticancer or antiviral effects [5, 6]. However, some studies have dealt with a negative physiological potential [7, 8]. Even though catechin and epicatechin have been analyzed by different analytical methods and tested in model systems, some aspects have not been investigated yet [9, 10]. Regarding their structure, catechin and epicatechin posess two chiral centers. Therefore, it is important to remember that the properties of chiral substances like (+)-catechin and (-)-epicatechin and their enantiomers depend on the conformation of the molecules (Figure 1).

Figure 1. Structures of the catechin /epicatechin enantiomers and procyanidin B2.<smiles>Oc1cc(O)c2c(c1)O[C@H](c1ccc(O)c(O)c1)C(O)C2</smiles>

$(+)$-Catechin $(2 \mathrm{R}, 3 \mathrm{~S})$<smiles>Oc1cc(O)c2c(c1)OC(c1ccc(O)c(O)c1)[C@H](O)C2</smiles>

(-)-Catechin (2S, 3R)<smiles>Oc1cc(O)c2c(c1)O[C@H](c1ccc(O)c(O)c1)[C@H](O)C2</smiles>

(-)-Epicatechin (2R, 3R)<smiles>Oc1cc(O)c2c(c1)OC(c1ccc(O)c(O)c1)[C@H](O)C2</smiles>

(+)-Epicatechin (2S, 3S)<smiles>Oc1cc(O)c2c(c1)O[C@H](c1ccc(O)c(O)c1)[C@H](O)C2c1c(O)cc(O)c2c1O[C@H](c1ccc(O)c(O)c1)[C@H](O)C2</smiles>

Procyanidin B2 
Consequently, some studies have shown totally different physiological effects of each enantiomer [10-13]. During biosynthesis catechin and epicatechin are predominantly synthesized as (+)-catechin and (-)-epicatechin [14]. The atypical flavan-3-ol enantiomers (-)-catechin and (+)-epicatechin, often referred to as ent-catechin and ent-epicatechin, rarely occur in nature and their presence seems to be induced by enzymatic action [15]. Vivanco and co-workers found racemic catechin in the roots of spotted knapweed (Centaurea maculosa, Lam.) and showing a high phytotoxic potential induced by (-)-catechin [16, 17]. Lately, the natural occurrence of all flavan-3-ol enantiomers, both (+/-)-catechin and (+/-)-epicatechin was described in guaraná seeds (Paullinia cupana var. sorbilis) [18]. Additionally, atypical flavan-3-ol enantiomers can also be formed by non-enzymatic action. High temperature and/or an increasing $\mathrm{pH}$ value are able to induce an epimerization reaction. The epimers of catechin and epicatechin participate in equilibrium reactions [19, 20]. Regarding processed food, monomeric flavan-3-ols could also be converted to their epimers. They were found in tea beverages and recently in cocoa and cocoa products [21, 22]. Donovan et al. separated both (+)- and (-)-catechin and (+)- and (-)-epicatechin by chiral HPLC [10]. In a previous attempt, we also tried to separate the flavan-3-ol enatiomers by chiral HPLC, applying a similar separation system, but the separation only succeeded for (+)- and (-)-catechin and failed for $(+)$ - and (-)-epicatechin [23]. Furthermore, technological methods to control the epimerization and the loss of the beneficial flavan-3-ols in cocoa products and other food by controlling the temperature and $\mathrm{pH}$ values during food processing have been developed [24-26].

Based on our earlier studies, we were interested in analyzing the atypical flavan-3-ol enantiomers focusing on their alteration in cocoa and cocoa products. We used chiral capillary electrophoresis (CCE) to separate the flavan-3-ols with cyclodextrins as chiral selectors [18]. Additionally we confirmed our results by isolating and purifying the flavan-3-ol monomers and performing LC-MS experiments.

\section{Results and Discussion}

Analysis of cocoa and cocoa products by CCE

Based on preliminary studies, we were able to separate both catechin and epicatechin into their two corresponding enantiomers using only one chiral selector added to the background electrolyte [18]. As shown in the electropherograms (see Figures 2-5), we were able to separate the flavan-3-ols in nearly 8 minutes. All samples were prepared under gentle and mild conditions, avoiding undesirable reactions of the analytes (see Experimental section). We applied the method to different cocoa and cocoa products. We thus confirmed that unfermented, dried, unroasted cocoa beans predominantely contain (-)- epicatechin and small amounts of (+)-catechin (see Figure 2). The analysis of different samples of roasted cocoa beans and commercial cocoa products like chocolate provides evidence for the occurrence of (-)-catechin besides (-)-epicatechin and (+)-catechin (see Figures 3 and 4). In cocoa powder and products containing cocoa powder, we even found increased levels of (-)-catechin (see Figure 5). 
Figure 2. Electropherogram showing the enantioselective separation of catechin and epicatechin in a) an extract of unfermented, dried, unroasted cocoa beans and b) isolated from this extract. 1: (-)-Epicatechin, 2: (+)-Catechin, P: Procyanidin B2. Experimental conditions: uncoated fused-silica capillary [60 cm (effective length $50 \mathrm{~cm}$ ) $\times 75 \mu \mathrm{m}$ I.D.]; electrolyte: $0.1 \mathrm{~mol} \cdot \mathrm{L}^{-1}$ borate buffer, $\mathrm{pH} 8.5$; chiral selector: $12.0 \mathrm{mmol} \cdot \mathrm{L}^{-1} \mathrm{HP}-\gamma$-CD; voltage: $18 \mathrm{kV}$; UV-Detection: $280 \mathrm{~nm}$; temperature: $20{ }^{\circ} \mathrm{C}$; injection: pressure, $3 \mathrm{~s}$, 0.3 p.s.i..

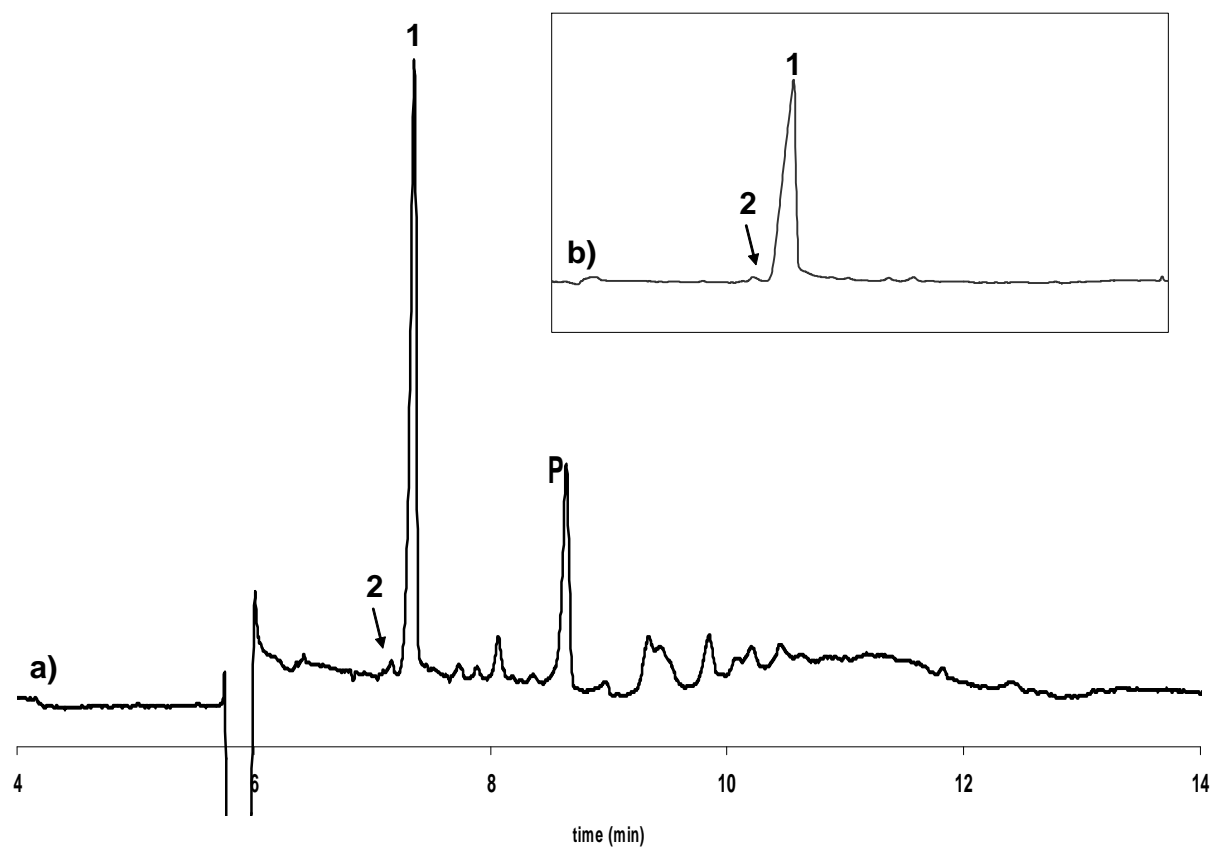

\section{Influence of temperature}

Our enantioselective analysis of cocoa and cocoa products showed that the occurrence of (-)-catechin is influenced by the cocoa processing conditions used. Our analysis showed that the first initial processing step for the formation of (-)-catechin is the roasting procedure (see Figure 3). Under the influence of high temperature (-)-epicatechin was converted into (-)-catechin, indicating an epimerization reaction. To confirm the influence of heat treatment during the roasting process we roasted unfermented cocoa beans which only contain (-)-epicatechin and (+)-catechin and undectectable levels of (-)-catechin (roasting conditions mentioned below). Analyzing the cocoa beans after the roasting procedure, we found (-)-catechin (results not shown). Therefore the occurrence of (-)-catechin in roasted cocoa beans is clearly induced by the roasting conditions. However, we could not exclude the possibility that in cocoa (-)-catechin additionally derives from the degradation of the procyanidins present. 
Figure 3. Electropherogram showing the enantioselective separation of catechin and epicatechin in a) an extract of roasted cocoa beans and b) isolated from this extract. 1: (-)-Epicatechin, 2: (+)-Catechin, 3: (-)-Catechin, P: Procyanidin B2. Experimental conditions as in Figure 2.

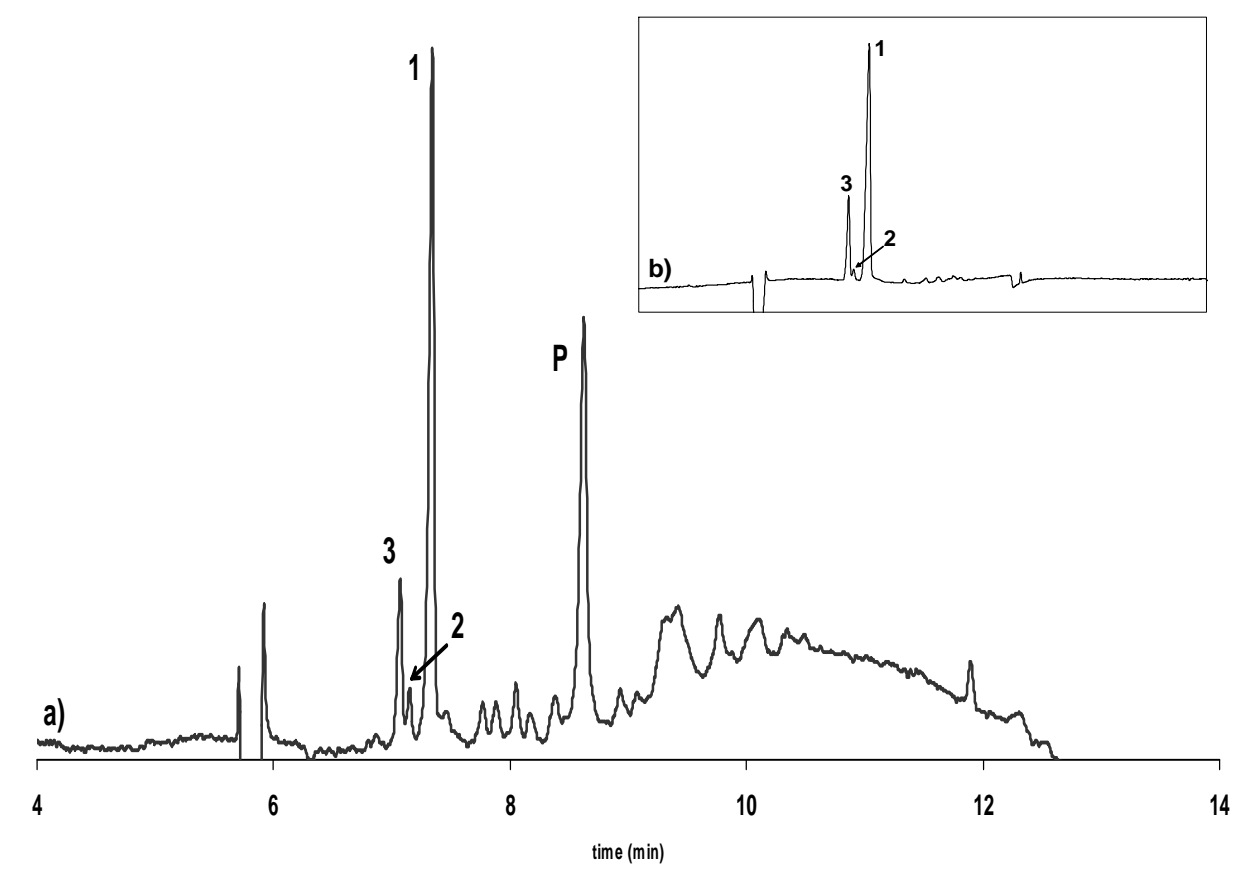

Analyzing cocoa products, like cocoa liquor or chocolate bars containing different levels of cocoa solids we also detected (-)-catechin (see Figure 3). After the roasting procedure the cocoa beans are ground to cocoa liquor and used as an ingredient for chocolate [27].

Another reason for the occurrence of (-)-catechin could be the epimerization reaction of the flavan-3-ols during sample preparation, which could be induced by high temperature and/or alkaline $\mathrm{pH}$ value. We could exclude these possibilities, because the whole sample preparation was accomplished at room temperature. The $\mathrm{pH}$ value of the methanolic/aqueous solution at the beginning of the cocoa extraction was 5.3 - 7.1. Additionally we couldn't detect any influence of oxygen or UV-light during the sample preparation. To confirm this, we conducted experiments under "extreme" conditions. A standard solution of (-)-epicatechin was aerated for 24 hours. After that time we analyzed the sample and could not find any epimerization products. To test the influence of daylight and UV-light, we prepared a (-)-epicatechin standard solution using the standard conditions. Another solution was irradiated by UV-light for 24 hours (experimental conditions mentioned below). Analyzing these samples by CCE, we could not find any epimerization products in the solution subjected by daylight conditions. On the other hand, in the UV irradiated solution we could find (-)catechin (results not shown). Therefore we can confidently exclude any influence of heat, light and oxygen to the epimerization reaction during sample preparation. 
Figure 4. Electropherogram showing the enantioselective separation of catechin and epicatechin in a) an extract of dark chocolate and b) isolated from this extract. 1: (-)-Epicatechin, 2: (+)-Catechin, 3: (-)-Catechin, P: Procyanidin B2. Experimental conditions as in Figure 2.

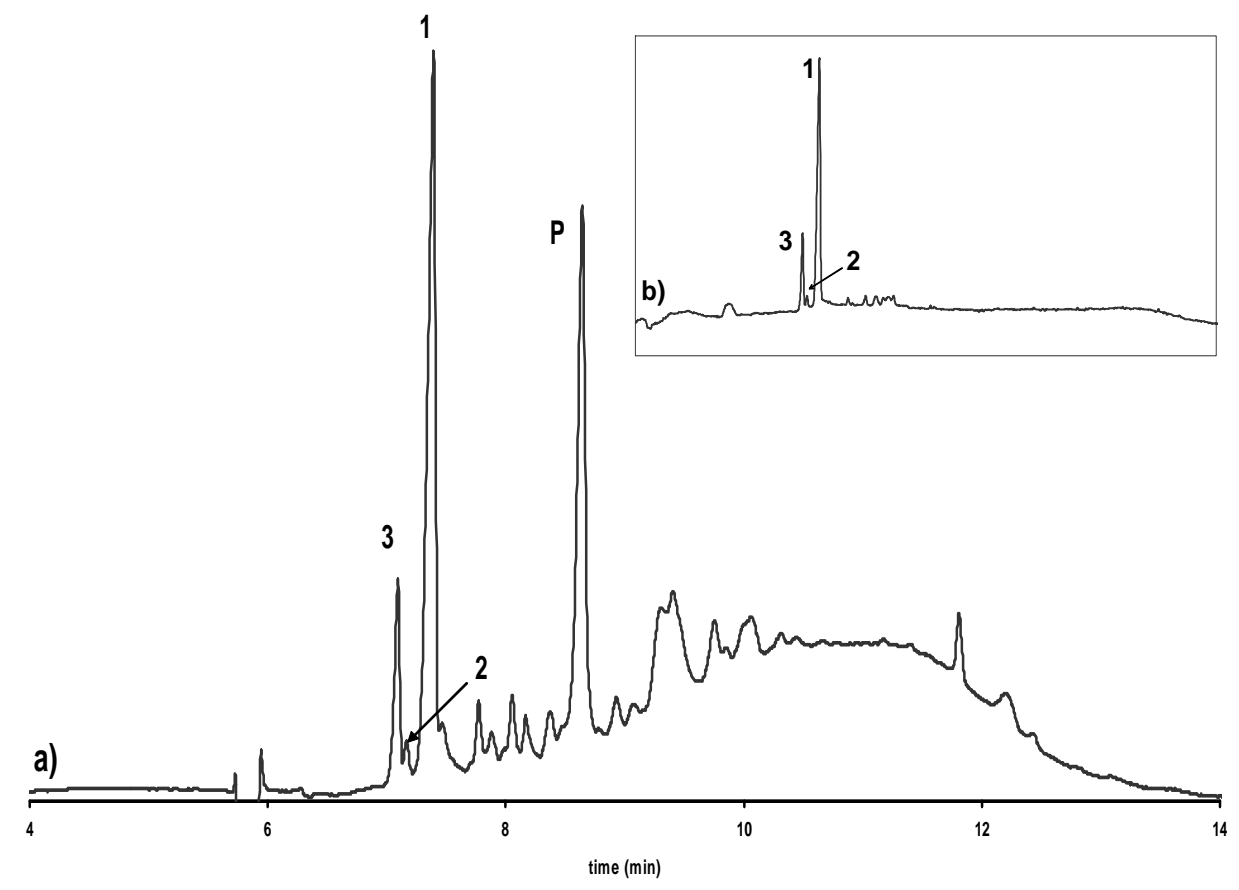

Influence of alkalization

As shown in Figure 5, we detected an increased level of (-)-catechin in cocoa powder and products containing cocoa powder, in contrast to chocolate samples. This fact is caused by the processing of cocoa powder. Cocoa liquor is alkalized to improve its technological and sensory qualities. Thus, it is typically treated for a certain period under basic $\mathrm{pH}$ and heat conditions and then neutralized to the desired $\mathrm{pH}$ value. The cocoa powder then can be used, for example, in instant chocolate drinks, commercial desserts or chocolate coatings. Under the alkaline conditions the epimerization reaction continues. After the alkalization of the cocoa liquor, it is subjected to pressure to separate the cocoa butter from the cocoa solids [27]. Perhaps the pressure applied (40-50 kPa) could also induce the epimerization reaction of the flavan-3-ols. We confirmed our results by alkalization experiments. Thus, we alkalized dried, unfermented, unroasted cocoa beans, which only contain (-)-epicatechin and $(+)$-catechin and no detectable (-)-catechin (experimental conditions mentioned below). The prepared extracts were analyzed by CCE. After this treatment we also found (-)-catechin in our samples. From this we infer that the occurrence of (-)-catechin in cocoa powder is first influenced by the roasting conditions and second by the alkalization process.

We could exclude any epimerization during the sample preparation and the CCE analysis. The enantioselective separation was performed with a borate buffer at $\mathrm{pH}$ 8.5. We separated the flavan-3-ol enantiomers during nearly 8 minutes and at a temperature of $20^{\circ} \mathrm{C}$. As shown in our previous studies, the analysis time used would be too short for any epimerization reactions to take place under the chosen conditions $[18,28]$. All results were confirmed by spiking experiments and comparison of the migration times to those obtained using standard solutions. 
Figure 5. Electropherogram showing the enantioselective separation of catechin and epicatechin in a) an extract of cocoa powder and b) isolated from this extract. 1: (-)-Epicatechin, 2: (+)-Catechin, 3: (-)-Catechin, P: Procyanidin B2. Experimental conditions as in Figure 2.

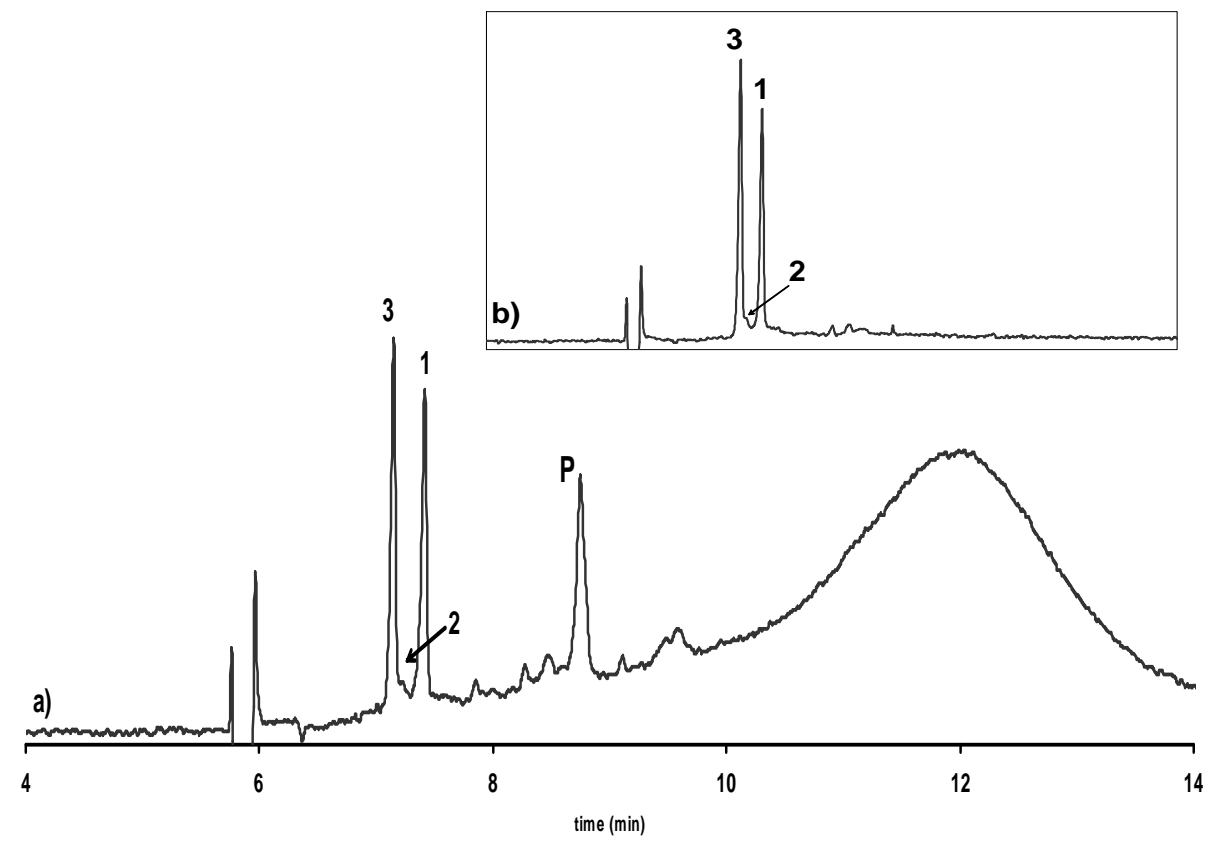

Isolation, purification and LC-MS analysis

The extracts from the different cocoa products show significant levels of procyanidins that are likely to comigrate with the monomeric flavanols in CCE separation. The monomeric flavanols were therefore isolated from the extracts and the identity and purity were confirmed by non-chiral HPLC-UV-iontrap MS analysis. After isolation and purification, no compounds are present anymore that would likely compromise CCE (see Figure 6).

\section{Comparison of the flavan-3-ol peak ratios}

Referring to former studies, the peak ratios of polyphenols can be much more interesting than the absolute amounts of flavan-3-ol enantiomers, because it is constant in a cultivar. The content of a particular substance may differ from year to year, but the peak ratios remain nearly constant. Therefore, during the last few years the use of peak ratios of polyphenolic substances has become more popular [29].

For a better comparison of our CCE results we calculated the peak ratio of the flavan-3-ol enantiomers of various cocoa beans, chocolate and cocoa powder samples. Table 1 shows the peak ratios of (-)-catechin and (-)-epicatechin calculated for different cocoa samples after enantioselective separation by CCE. During the processing of cocoa, degradation of catechin and epicatechin is enormous compared to their levels in unfermented cocoa beans, which is caused by the fermentation and the roasting procedure [30]. Additionally, the major flavan-3-ol enantiomer, (-)-epicatechin is epimerized to (-)-catechin. In unfermented, dried, unroasted cocoa beans we were unable to identify 
(-)-catechin by CCE. However, we found (-)-catechin in roasted cocoa beans and their subsequent products, like cocoa liquor and chocolate. In these products the peak ratios of (-)-catechin and (-)-epicatechin are in a similar range. It can therefore be concluded that one of the main reasons for the epimerization of (-)-epicatechin to (-)-catechin is the roasting procedure. The ratios of the non alkalized cocoa powders are different. By processing non-alkalized cocoa powder the cocoa liquor will be subjected to heat and pressure to extract the cocoa butter from the cocoa solids [27]. We can not exclude the possibility that the epimerization reaction continues after this. Comparing the (-)-catechin and (-)-epicatechin peak ratios of alkalized cocoa powder we observed enormous differences (see Table 1). As mentioned above during the alkalization process the epimerization reaction continues.

Figure 6. MS spectra (averaged over the retention time frame of the flavan-3-ols, 20-35 min) of a) the raw cocoa bean extract and b) the isolated and purified flavan-3-ols from this extract. The signals in the extract spectrum correspond to procyanidins. The small signals in the isolated flavanols correspond to ionization-induced adducts and fragments and background noise.

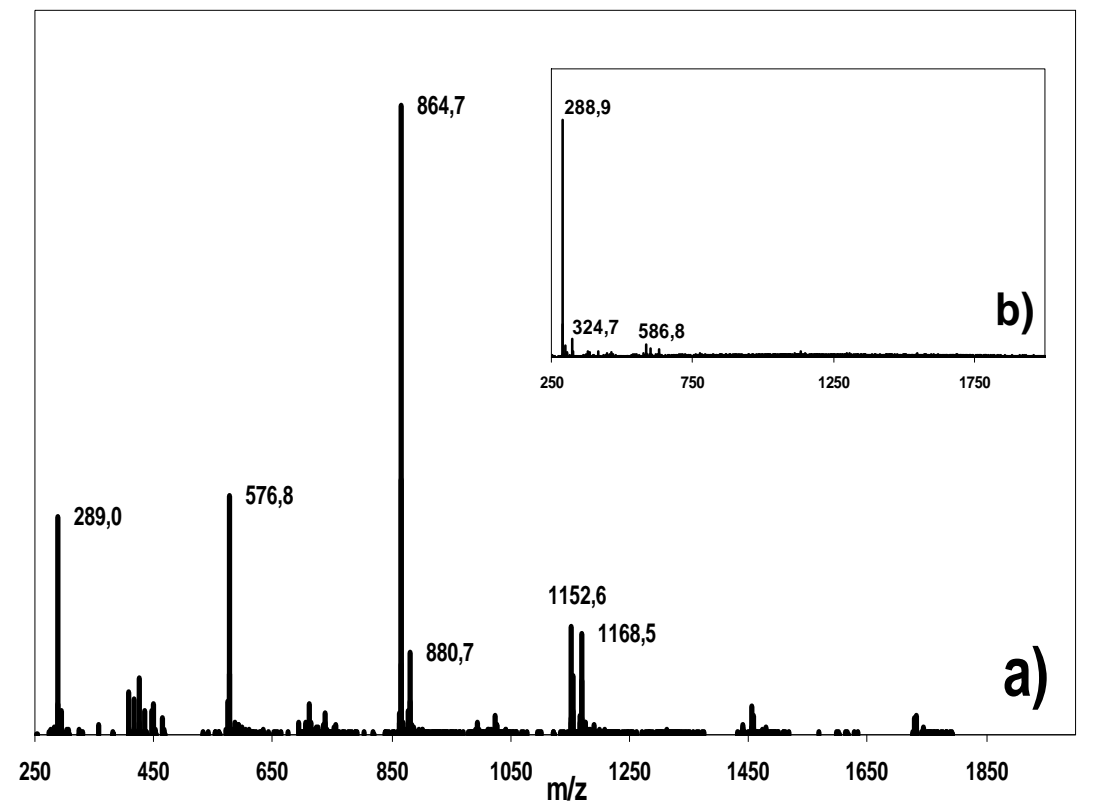

In contrast to (-)-catechin, which displayed increasing levels during cocoa processing, $(+)$-epicatechin was not detected by CCE in the analyzed samples, which would be expected to occur as a product of the epimerization of $(+)$-catechin. This finding may be due to several reasons: (1) $(+)$-catechin is only a minor genuine component of the natural flavanols in cocoa in contrast to the dominating (-)-epicatechin. Consequently, in comparison to the (-)-catechin levels, only small amounts of (+)-epicatechin would likely be formed during the epimerization reaction; (2) generally, the sensitivity of the detection used in CCE is limited and moreover the small amounts of (+)-epicatechin are not sufficiently separated from the close eluting predominant (-)-epicatechin peak; (3) the transoid structure of the substituents at C-2 and C-3 in catechin is thermodynamically favoured over the cisoid structure in epicatechin (see Figure 1). The formation of (-)-catechin from (-)-epicatechin is therefore 
likely favoured in comparison to the formation of (+)-epicatechin from (+)-catechin [31]. These factors might all together account for this finding.

Table 1. Peak ratios of (-)-catechin and (-)-epicatechin for the enantioselective separation of catechin and epicatechin in different cocoa and cocoa product extracts.

\begin{tabular}{lc} 
Sample & $\begin{array}{c}\text { CCE Peak ratios of } \\
\text { (-)-Catechin/(-)-Epicatechin }\end{array}$ \\
\hline Raw, unfermented cocoa beans & $-*$ \\
\hline Roasted cocoa beans & $18: 82$ \\
Roasted cocoa beans (nibs) & $19: 81$ \\
Cocoa liquor (non-alkalized) & $18: 82$ \\
Chocolate (85\% cocoa solids) & $18: 82$ \\
\hline Cocoa Powder (non-alkalized) & $24: 76$ \\
Cocoa Powder (non- alkalized) & $35: 65$ \\
\hline Cocoa Powder (alkalized)** & $42: 58$ \\
Cocoa Powder (alkalized) $* *$ & $65: 35$ \\
\hline & $*$ undectectable level of (-)-catechin \\
& $* *$ grade of alkalization not known \\
\end{tabular}

\section{Conclusions}

Cocoa and chocolate samples were analyzed by CCE using the enantioseparation of catechin and epicatechin. (-)-Catechin was found among (-)-epicatechin and (+)-catechin as a result of an epimerization reaction. (+)-Epicatechin, however, could not be determined. It was found that (-)-catechin is formed during the roasting process and especially during the alkalization of the cocoa powder. The results were confirmed by isolating and analyzing the monomeric flavan-3-ols by solid-phase-extraction and LC-MS, respectively. We observed a degradation of (-)-epicatechin during the manufacturing process. Additionally (-)-epicatechin levels decreased caused by its epimerization to (-)-catechin. Comparing the differences of peak ratios between (-)-catechin and (-)-epicatechin, we could characterize the influence of roasting and particularly alkalization.

\section{Outlook}

The epimerization of flavan-3-ol enatiomers should be intensively studied with regard to the technological parameters during the roasting and alkalization process. Furthermore, an appropriate chiral HPLC method should be applied, verifying the occurrence of (+)-epicatechin, which is likely formed from (+)-catechin. 


\section{Experimental}

\section{General}

Standards of (+)-catechin, (-)-catechin, (+)-epicatechin, and (-)-epicatechin were obtained from Sigma (St. Louis, MO, USA). Anhydrous sodium tetraborate, boric acid, sodium hydroxide, (2-hydroxypropyl)- $\gamma$-cyclodextrin, and sodium dihydrophosphate were purchased from Fluka Chemie (Buchs, Switzerland), and dimethylsulfoxide from Merck (Darmstadt, Germany). Methanol and acetonitrile were purchased from Fisher Scientific (Loughborough, UK). For unfermented, dried, unroasted beans, cocoa fruits were purchased from a Brasilian supermarket (Campinas/Sao Paolo, Brazil) and the raw beans removed manually and sundried. Roasted cocoa beans, chocolate and cocoa products were bought from a local supermarket. Catechin standards were diluted in dimethylsulfoxide (DMSO) to a concentration of $0.5 \mathrm{mg} \cdot \mathrm{mL}^{-1}$ and were stored at $8{ }^{\circ} \mathrm{C}$. The background electrolyte (BGE) was prepared by dissolving appropriate amounts of boric acid and anhydrous sodium tetraborate adjusted to desired $\mathrm{pH}$ values by using $\mathrm{NaOH}$ [32]. The purified water we used in this study was produced by an Elgastat UHQ II (Elga Ltd., Buds, UK). The chiral selectors were dissolved to different concentrations in the running buffer. Before use, the buffer solutions were filtered through a $0.45 \mu \mathrm{m}$ regenerated cellulose syringe filter (IVA, Meerbusch, Germany). The cocoa beans, chocolate samples, and cocoa product samples were ground in a pebble mill (MM Retsch, Haan, Germany). An amount (500 mg) was mixed with $\mathrm{MeOH}+$ purified water (30 mL, 80:20, v/v) and stirred for 2 hours. Then methanol was evaporated at room temperature at ambient pressure in a fume hood. The residue was diluted in purified water (30 mL), centrifuged at $10000 \mathrm{rpm}$ for $15 \mathrm{~min}$ in a Biofuge Stratos (Heraeus Holding GmbH, Hanau, Germany) and filtered through a folded filter paper (Schleicher and Schuell Microscience GmbH, Dassel, Germany). The filtrate was purified and concentrated by solid phase extraction using a previously published method with a $6 \mathrm{~mL}$ size Chromabond PA cartridge (Macherey-Nagel, Düren, Germany) packed with $1000 \mathrm{mg}$ of polyamide. The cartridge was conditioned with DMSO+formic acid (2 mL, 99:1, v/v) for $10 \mathrm{~min}$ and washed with purified water $(10 \mathrm{~mL})$. An aliquot of the above mentioned filtrate $(30 \mathrm{~mL})$ was loaded onto the SPE cartridge quantitatively and the cartridge was washed with purified water $(20 \mathrm{~mL})$ and DMSO+formic acid (1.5 mL, 99:1, v/v). Finally, the adsorbed analytes were eluted with the latter solvent mixture $(2.0 \mathrm{~mL})$ and the eluate was used for the CCE [33].

\section{Roasting, alkalization, temperature and UV experiments}

Roasting and alkalization experiments were performed using unfermented, dried, unroasted cocoa beans. The beans were roasted in a Memmert UL 50 drying oven (Memmert, Schwalbach, Germany) at $120{ }^{\circ} \mathrm{C}$ for 2 hours or at $160{ }^{\circ} \mathrm{C}$ for 2.5 hours. After that time, the preparation of the beans followed the sample preparation mentioned above. For the alkalization experiments, the ground beans were mixed with $\mathrm{MeOH}+$ purified water $(30 \mathrm{~mL}, 80: 20, \mathrm{v} / \mathrm{v}$ ) for 2 hours. The methanol was evaporated at room temperature. The residue was diluted with $10 \mathrm{~mol} \cdot \mathrm{L}^{-1} \mathrm{Na}_{2} \mathrm{CO}_{3}$ solution (30 mL, $\mathrm{pH} 9.6$ ), centrifuged at $10000 \mathrm{rpm}$ for $15 \mathrm{~min}$ in the Biofuge Stratos and filtered through a folded filter paper. Then the solution was heated in a water bath for 1 hour at $75^{\circ} \mathrm{C}$. After that, the solution was cooled 
down to room temperature, neutralized to $\mathrm{pH} 6.1$ with $0.1 \mathrm{~mol} \cdot \mathrm{L}^{-1}$ citric acid and purified and concentrated by the solid phase extraction procedure described above. For the temperature experiments, a standard solution of (-)-epicatechin $\left(0.5 \mathrm{mg} \cdot \mathrm{mL}^{-1}\right)$ was stored in a beaker at room temperature for 24 hours. After that time, the solution was analyzed by CCE. For the daylight and UVexperiments a standard solution of (-)-epicatechin $\left(0.5 \mathrm{mg} \cdot \mathrm{mL}^{-1}\right)$ was exposed to daylight for 24 hours and another was irradiated with UV-light (254, $280 \mathrm{~nm}$ ) using a "Fluotest Universal” (Original Hanau Quarzlampen GmbH, Hanau, Germany) for 24 hours. After that time, the solution was analyzed by CCE.

\section{Isolation and purification of monomeric flavan-3-ols from extracts}

Isolation and purification of the monomeric flavan-3-ols from cocoa and chocolate extracts was performed using $3 \mathrm{~mL}$ size ChromaBond solid phase extraction cartridges packed with $500 \mathrm{mg}$ of polyamide (Macherey-Nagel, Düren, Germany), according to a previously described method with slight modifications [18]. The cartridge was conditioned with DMSO+formic acid (2 mL, 99:1, v/v) and purified water $(5 \mathrm{~mL})$. The extract $(500 \mu \mathrm{L})$ was dissolved in purified water $(9.5 \mathrm{~mL})$, loaded onto the cartridge and the polyamide was then washed with purified water $(10 \mathrm{~mL})$ and $\mathrm{MeOH}(2 \mathrm{~mL})$. The monomeric flavan-3-ols were recovered from the SPE cartridge by elution with additional $\mathrm{MeOH}$ (3 mL). The solvent was evaporated in vacuo and the compounds dissolved in DMSO+formic acid (500 $\mu \mathrm{L}, 99: 1, \mathrm{v} / \mathrm{v}$ ) prior to analysis. The extracts as well as the isolated monomeric flavanols were analyzed by HPLC-UV-ion trap MS to verify the identity of the flavan-3-ols and verify the purity. Subsequently, the isolated flavanols were subjected to CCE.

\section{HPLC- MS analysis}

Individual polyphenols were identified by multi step mass spectrometric fragmentation after HPLC separation and UV-VIS diode array detection. A Summit HPLC System (Dionex, Idstein, Germany) equipped with an Aqua $3 \mu \mathrm{m} \mathrm{C18,} 150 \mathrm{~mm} \times 2 \mathrm{~mm}$ I.D. analytical column (Phenomenex, Aschaffenburg, Germany) kept at $35^{\circ} \mathrm{C}$ was used. $1 \%$ Acetic acid in purified water (mobile phase A) and $1 \%$ acetic acid in acetonitrile (mobile phase B) were used at $300 \mu \mathrm{L} \cdot \mathrm{min}^{-1}$ starting at $0 \%$ B with a linear gradient to $30 \%$ B after 60 minutes followed by washing (100\% B for $10 \mathrm{~min}$ ) and reequilibration ( $0 \% \mathrm{~B}$ for $10 \mathrm{~min}) .5 \mu \mathrm{L}$ of each sample were injected and the chromatograms monitored at 200-595 $\mathrm{nm}$.

An LCQ Classic ion-trap mass spectrometer (Thermo Fisher Scientific, Dreieich, Germany) with an ESI-interface and a metal needle kit was operated in the negative mode as published earlier [18, 33]. The phenolic compounds were detected in their deprotonated form as the quasimolecular ion [M$\mathrm{H}]^{-}$one mass unit below their molecular mass. Identification of individual compounds was conducted by comparison of their UV-spectra and ion trap fragmentation patterns with a self prepared library from standard substances and known compounds as described in detail previously [33-35]. 


\section{Chiral capillary electrophoretic analysis}

All chiral separations were carried out on a Beckman P/ACE MDQ capillary electrophoretic system (Beckman Instruments, Fullerton, CA, USA) equipped with a photo diode array detection system. The scan range was 190 to $350 \mathrm{~nm}$. All electropherograms were recorded at $280 \mathrm{~nm}$. Data was processed on an IBM personal computer with Beckman 32 Karat software version 7.0. The identification of the analytes was achieved comparing the migration times and the UV-spectra of the flavan-3-ols and by spiking with standard solutions of the single enantiomers. Uncoated fused-silica capillaries of $375 \mu \mathrm{m}$ O.D. $\times 75 \mu \mathrm{m}$ I.D. were obtained from Beckman and cut to $60.2 \mathrm{~cm}$ (effective length $50 \mathrm{~cm}$ ). The capillary was first washed by 20 p.s.i. pressure with $0.25 \mathrm{~mol} \cdot \mathrm{L}^{-1} \mathrm{NaOH}$ for $20 \mathrm{~min}$ and finally with purified water for $10 \mathrm{~min}$; between each run with $0.25 \mathrm{~mol} \cdot \mathrm{L}^{-1} \mathrm{NaOH}$ for $3 \mathrm{~min}$, with purified water for $2 \mathrm{~min}$, and equilibrated with the running buffer for $5 \mathrm{~min}$; for the overnight storage with $0.25 \mathrm{~mol} \mathrm{~L}^{-1} \mathrm{NaOH}$ for $5 \mathrm{~min}$, with purified water for $10 \mathrm{~min}$, and an air stream for 3 minutes to remove the liquid in the capillary. The capillary temperature was set to $20{ }^{\circ} \mathrm{C}$ and the applied voltage was $18 \mathrm{kV}$. Samples were injected hydrodynamically ( 0.3 p.s.i.) for $3 \mathrm{~s}$. We injected purified water for 2 s (0.2 p.s.i.) after and before injecting the sample into the capillary. In this way the contamination of the sample vial with small amounts of the running buffer was avoided. For every pair of buffer we used a separate vial of purified water. This procedure has no negative effect on the chiral separation system, but solves the problem of sample degradation as studied previously [23].

\section{Analytical characteristics (CCE)}

The linearity of the CCE method in the $20-200 \mu \mathrm{g} \cdot \mathrm{mL}^{-1}$ range was demonstrated by standard curves for $(+)$-catechin, (-)-catechin and (-)-epicatechin $\left(r^{2}>0.999\right)$. It has to be mentioned that to achieve a sufficient peak resolution in chiral separations by capillary electrophoresis, the peak height of a chiral substance should be less than $5 \mathrm{mAU}$ [18]. The reproducibility of the migration time was investigated for (+)-catechin (RSD $0.8 \%$ intra-day (n=3), RSD $0.97 \%$ inter-day (n=9)) and (-)-epicatechin (RSD 0.77\% intra-day ( $\mathrm{n}=3$ ), RSD 0.99\% inter-day ( $\mathrm{n}=9$ )). Detection limits: (defined as a signal-to-noise ratio of 3) limit of detection $=5 \mu \mathrm{g} \cdot \mathrm{mL}^{-1}$ and limit of quantification $=10 \mu \mathrm{g} \cdot \mathrm{mL}^{-1}$ (defined as a signal-to-noise ratio of 6) for (+)-catechin, (-)-catechin and (-)-epicatechin.

\section{Acknowledgements}

The authors acknowledge PD Dr. Friedhelm Marx and Dr. Roberta Rodrigues, University of Bonn, for the kind gift of raw, dried cocoa beans.

\section{References}

1. Arts, I.C.W.; van de Putte, B.; Hollman, P.C.H. Catechin contents of foods commonly consumed in The Netherlands. 1. Fruits, vegetables, staple foods, and processed foods. J. Agric. Food Chem. 2000, 48, 1746-1751. 
2. Harnley, J.M.; Doherty, R.F.; Beecher, G.R.; Holden, J.M.; Haytowitz, D.B.; Bhagwat; S.; Gebhardt, S. Flavonoid content of U.S. fruits, vegetables, and nuts. J. Agric. Food Chem. 2006, 54, 9966-9977.

3. Hooper, L.; Cassidy, A. A review of the health potential of bioactive compounds. J. Sci. Food Agric. 2006, 86, 1805-1813.

4. Treutter, D. Significane of flavonoids in plant resistance: A review. Environ. Chem. Lett. 2006, 4, 147-157.

5. Friedman, M. Overview of antibacterial, antitoxin, antiviral, and antifungal activities of tea flavonoids and teas. Mol. Nutr. Food Res. 2006, 51, 116-134.

6. Kermez, Z.; Chetrit, D.; Shoseyov, O.; Regev-Shosani, G. Protection of lipids from oxidation by epicatechin, trans-resveratrol, and gallic and caffeic acids in intestinal model systems J. Agric. Food Chem. 2006, 54, 10288-10293.

7. Murphy, K.J.; Chronopoulos, A.K.; Singh, I.; Francis, M.A.; Moriarty, H.; Pike, M.J.; Turner, A.H.; Mann, N.J.; Sinclair, A.J. Dietary flavanols and procyanidin oligomers from cocoa (Theobroma cocoa) inhibit platelet function. Am. J. Clin. Nutr. 2003, 77, 1466-1473.

8. Hurell, R.F.; Reddy, M.; Cook, J.D. Inhibition of non-haem iron aborption in man by polyphenolic-containing beverages. Brit. J. Nutr. 1999, 81, 289-295.

9. Vinson, J.A.; Proch, J.; Bose, P.; Muchler, S.; Taffera, P.; Shuta, D.; Samman, N.; Agbor, G.A. Chocolate is a powerfull ex vivo and in vivo antioxidant, an antiathereosclerotic agent in animal model, and a significant contributor to antioxidants in european and american diets. J. Agric. Food Chem. 2006, 54, 8071-8076.

10. Donovan, J.L.; Crespy, V.; Oliveira, M.; Cooper K.A.; Gibson, B.B.; Williamson, G. (+)-Catechin is more bioavailable than (-)-catechin: Relevance to the bioavailability of catechin from cocoa. Free Radical Res. 2006, 40, 1029-1034.

11. Nyfeler, F.; Moser, U.K.; Walter, P. Stereospecific effects of (+)- and (-)-catechin on glycogen metabolism in isolated rat hepatocytes. Biochim. Biophys. Acta 1983, 763, 50-57.

12. Cao, Y., Liang, R.; Yang, B.; Wang, L.; Yang, Q.; Wang, Y. Protection of (-)-catechin gallate and (+)-epicatechin on xanthine-xanthine oxidase system injury in cultered cardiomyocytes. Zhongguo Shiyan Fangjixue Zazhi 2006, 12, 36-38.

13. Cho, S.Y.; Park, P.J.; Shin, H.J.; Kim, Y.K.; Shin, D.W.; Shin, E.S.; Lee, H.H.; Lee, B.G.; Baik, J.H.; Lee, T.R. (-)-Catechin suppresses expression of Kruppel-like factor 7 and increases expression and secretion of adiponectin protein in 3T3-L1 cells. Am. J. Physiol. Endocrinol. Metab. 2007, 292, E1166-E1172.

14. Pfeiffer, J.; Kühnel, C.; Brandt, J.; Duy, D.; Punyasiri, P.A.N.; Forkmann, G.; Fischer, T.C. Biosynthesis of flavan-3-ols by leucoanthocyanidin 4-reductase and anthocyanidin reductase in leaves of grape (Vitis vinifera L.), apple (Malus $x$ domestica Borkh.) and other crops. Plant Physiol. Bioch. 2006, 44, 323-334.

15. Ellis, C.J.; Yeap Foo L.; Porter, L.J. Enantiomerism: A characteristic of the proanthocyanidin chemistry of the Monocotyledonae. Phytochem. 1983, 22, 483-487.

16. Blair, A.C.; Nissen, S.J.; Brunk, G.R.; Hufbauer, R.A. A lack of evidence for an ecological role of the putative allelochemical (+/-)-catechin in spotted knapweed invasion success. J. Chem. Ecol. 2006, 32, 2327- 2331. 
17. Prithiviraj, B.; Perry, L.G.; Badri, D.V.; Vivanco, J. M. Chemical facilitation and induced pathogen resistance mediated by root-secreted phytotoxin. New Phytol. 2007, 173, 852-860.

18. Kofink, M.; Papagiannopoulos, M.; Galensa, R. Enantioseparation of catechin and epicatechin in plant food by chiral capillary electrophoresis. Eur. Food Res. Technol. 2007, 225, 569-577.

19. Haslam, E. In The flavonoids; Harborne, J.B., Ed.; Chapman and Hall: London, 1975; Chapter 10, p 550-553

20. Kennedy, J.A.; Munro, M.H.G.; Powell, H. K. J.; Porter, L.J.; Yeap Foo, L.; The protonation reactions of catechin and epicatechin and related compounds. Aust. J. Chem. 1984, 37, 885-892.

21. Kodama, S.; Yamamoto, A.; Akinobu, M.; Yanai, H. Direct enatioseparation of catechin and epicatechin in tea drinks by 6-O- $\alpha$-D-glucosyl- $\beta$-cyclodextrin-modified micellar electrokinetic chromatography. Electrophoresis 2004, 25, 2892-2898.

22. Gotti, R.; Furlanetto, S.; Pinzauti, S.; Cavrini, V. Analysis of catechins in Theobroma cacoa beans by cyclodextrin-modified micellar electrokinetic chromatography. J. Chromatogr. A 2006, 1112, 345-352.

23. Gel-Moreto, N. Enantiomerentrennung von Polyphenolen in Citrus mittels Kapillarelektrophorese (CE) und Hochleistungsflüssigkeitschromatographie (HPLC). PhD Thesis, University of Bonn, 2003.

24. Hammerstone JR., J.F.; Acquarone, V. Process for controlling the isomerization of (-)-epicatechin and (+)-catechin in edible products. U.S. Pat. Appl. 2007/0003640, 2007.

25. Woefel, K.A.; Robbins, R.J. Products containing polyphenols. WO 2007/002883, 2007.

26. Hammerstone JR., J.F.; Acquarone, V. Heat-processed products having altered monomer profiles and processes for controlling the epimerization of (-)-epicatechin and (+)-catechin in the products. WO 2007/002852, 2007.

27. Beckett, S.T. The science of chocolate; Royal Society of Chemistry: Cambridge, 2000; pp. 31-47.

28. Komatsu, Y.; Suematsu, S.; Hisanobu, Y.; Saigo, H.; Matsuda, R.; Hara, K. Effects of pH and temperature on reaction kinetics of catechins in green tea infusion. Biosci. Biotech. Biochem. 1993, 57(6), 907-910.

29. Zimmermann, B.F.; Galensa, R. One for all-all for one: Proof of authenticity and tracing of foods with flavonoids. Analysis of proanthocyanidins in barley and malt. Eur. Food Res. Technol. 2006, 24, 385-393.

30. Wollgast, J.; Anklam, E. Review on polyphenols in Theobroma cacao: Changes in composition during the manufacture of chocolate and methodology for identification and quantification. Food Res. Int. 2000, 33, 423-447

31. Kiatgrajai, P.; Wellons, J.D.; Gollob, L.; White, J.D. Kinetics of epimerization of (+)-catechin and its rearrangement to catechinic acid. J. Org. Chem. 1982, 47, 2910-2912.

32. Wätzig, H.; Degenhardt, M.; Kunkel, A. Strategies for capillary electrophoresis: Method development and validation for pharmaceutical and biological applications. Electrophoresis 1998, 19, 2695-2752.

33. Papagiannopoulos, M.; Wollseifen, H.R.; Mellenthin, A.; Haber, B.; Galensa, R. Identification and quantification of polyphenols in Carob Fruits (Ceratonia siliqua L.) and derived products by HPLC-UV-ESI/MS ${ }^{\mathrm{n}}$. J. Agric. Food Chem. 2004, 52, 3784-3791. 
34. Friedrich, W.; Eberhardt, A.; Galensa, R. Investigations of proanthocyanidins by HPLC with electrospray ionization mass spectrometry. Eur. Food Res. Technol. 2000, 211, 56-64.

35. Papagiannopoulos, M.; Zimmermann, B.; Mellenthin, A.; Krappe, M.; Maio, G.; Galensa, R. Online coupling of pressurized liquid extraction, solid-phase-extraction and high-performance liquid chromatography for automated analysis of proanthocyanidins in malt. J. Chromatogr. A 2002, 958, 9-16.

Sample Availability: Samples of the compounds are available from the corresponding author.

(C) 2007 by MDPI (http://www.mdpi.org). Reproduction is permitted for noncommercial purposes. 